\title{
Espacios, relaciones y significados del sexo anónimo entre varones en México. Figuraciones entre lo alternativo a lo normativizado
}

\author{
Luis Arturo Sánchez Domínguez \\ Escuela Nacional de Antropología e Historia, México \\ luisarturos@yahoo.com
}

\section{Resumen}

En este texto se presentan y analizan algunos espacios, relaciones y significados del sexo anónimo entre varones en México, particularmente de su ciudad capital. El objetivo no solo es descriptivo, sino analítico, tratando de entender cómo pueden coexistir dos significados en apariencia contradictorios: por un lado, la alternativa transgresora de las normas morales y sexuales; y por el otro, el cómo se reafirma con ellas un orden heteronormativo, en donde placer y poder se hacen presentes en formas de dominación, abuso, discriminación o transgresión. Se trata de una interpretación de esta práctica desde las consideraciones sociales, la historia local y la subjetividad individual, que no pretende mostrar un significado detrás del sexo anónimo de los hombres homosexuales en México, sino reflexionar sobre precisamente, el complejo universo de significados que lo determinan.

Se pretende contribuir con la discusión del tema, aportando datos y experiencias de investigación, en donde es necesario replantear el llamado "modelo dominante de la comprensión del homoerotismo entre varones" (Véase Núñez Noriega, 2001), pues no se trata de un fenómeno único o idéntico, sino de uno con gran variedad de posibles lugares de encuentro, muy diversas prácticas entre sujetos también diversos, que interactúan bajo referentes o imaginarios igualmente variados, pero que en conjunto, crean o recrean interpretaciones compartidas. Bajo esta perspectiva dinámica de la significación, retomaré el concepto de "figuraciones" propuesto por Norbert Elías (1999), para destacar los procesos que están detrás de esta práctica.

Palabras clave: ligue, homosexualidad, sexo anónimo, cruising. 


\title{
Spaces, relationships and meanings of anonymous sex between men in Mexico. Figurations between the alternative to the standardized
}

\begin{abstract}
This text presents and analyzes some spaces, relationships and meanings of anonymous sex between men in Mexico, particularly in their capital city. The objective is not only descriptive, but analytical, trying to understand how two apparently contradictory meanings can coexist: on the one hand, the transgressive alternative of moral and sexual norms; and on the other, how heteronormative order is reaffirmed with them, where pleasure and power are present in forms of domination, abuse, discrimination or transgression. It is an interpretation of this practice from social considerations, local history and individual subjectivity, which is not intended to show a meaning behind the anonymous sex of homosexual men in Mexico, but to reflect on precisely the complex universe of meanings that determine.

It is intended to contribute to the discussion of the topic, providing data and research experiences, where it is necessary to rethink the so-called "dominant model of the understanding of homoeroticism among men" (See Núñez Noriega, 2001), since it is not a question of unique or identical phenomenon, but of one with a great variety of possible meeting places, very diverse practices among also diverse subjects, that interact under equally varied referents or imaginary, but that together, create or recreate shared interpretations. Under this dynamic perspective of significance, I will resume the concept of "figurations" proposed by Norbert Elías (1999), to highlight the processes in this practice.
\end{abstract}

Keywords: flirt, homosexuality, anonymous sex, cruising.

Fecha de recepción: 28 de junio de 2019.

Fecha de aprobación: 04 de febrero de 2020. 


\section{Introducción}

En nuestros días, y aún después de los cambios en torno a las libertades homosexuales, el sexo anónimo entre varones sigue siendo una práctica controvertida a partir de la hegemónica "heterosexualidad obligatoria" y el pensamiento patriarcal. La reflexión sobre estos espacios la inicié con la tesis de maestría en Antropología Social (2000-2003). A partir de ese trabajo fui descubriendo la geografía para los encuentros anónimos de la ciudad de México, gracias a la observación participante y la realización de entrevistas. Desde entonces he seguido acudiendo a diferentes sitios o recorridos urbanos del encuentro sexual anónimo, tanto en la ciudad de México como en otras ciudades de país, estas experiencias directas se han complementado con una gran cantidad de literatura que aborda el tema desde variadas perspectivas metodológicas y en conjunto nos ofrecen un buen número de testimonios.

En los tres apartados presentados se recurre como elemento metodológico al concepto de "Figuraciones" pues estas prácticas están marcadas tanto por estructuras externas o coercitivas, como filtros individuales de las situaciones: socio genética y psicogenética, mismos que dan sentido estructurante a los significados y se reflejan en las formas precisas de interacción e interdependencia recíproca (Elías, 1999), es decir: figuraciones entre lo alternativo y lo normativizado.

\section{La sociogénesis del sexo anónimo.}

Las prácticas homoeróticas y el sexo casual anónimo, forman parte de la realidad cotidiana de las principales ciudades mexicana, pero a lo largo de la historia, estas prácticas han sido el resultado del desarrollo de la modernidad y un paulatino proceso civilizatorio en que fue modificando el disciplinamiento corporal, el control de la sexualidad, la separación de lo público y lo privado y la aparición del sujeto homosexual. Se trata de un universo complejo de cambios que de forma directa o indirecta revelan acontecimientos históricos y culturales tanto globales como locales que es necesario tener en cuenta para comprender el fenómeno del sexo casual y sus múltiples significados.

Esa separación entre lo público y lo privado, lo correcto y lo vergonzoso, lo moral e inmoral, lo sano y lo enfermo, construye un sistema de valores sociales que poco a poco fueron configurando 
las concepciones de la práctica homosexual, pero ni en tiempo, espacio, materialidad o significado, han sido igual para las distintas épocas, sociedades y culturas (Véase Cardín, 1989; Herdt, 1997 y, Balbuena y Ovalle, 2013); no obstante, nos hacen pensar en prácticas en las que el placer, el poder o el deseo estuvieron presentes y que pueden ser referente para entender el resultado complejo de las relaciones actuales.

De un fascinante despliegue voyeurístico y linguófilo, los y las antropólogas (y otros lectores) hemos aprendido que entre los Sambia de Nueva Guinea el fellatio entre varones de diferentes edades es parte de un sistema ritual de masculinización (Herdt, 1981); que en la Grecia antigua la relación homoerótica se encontraba estratificada por edad y concebida en términos pedagógico políticos (Foucault, 1986); que en algunas tribus norteamericanas los varones con preferencias homoeróticas y "andróginas" tienen un estatus especial que les permite "matrimoniarse" con otro varón y desempeñar actividades religiosas y ceremoniales importantes en su sociedad (Williams, 1986); o que en Nicaragua un hombre puede tener relaciones sexuales con otro hombre sin ser estigmatizado, siempre y cuando desempeñe en la relación anal el papel "activo" de penetrador (Lancaster, 1992, citado por Núñez Noriega, 2001:16-17).

En el caso de México, el antecedente de la vida homosexual moderna se dio durante el porfiriato en 1901, con la redada de los 41en la calle de Esequiel Montes, en la actual colonia Tabacalera. Se trataba de una fiesta privada de la alta burguesía en la que hombres bailaban con hombres vestidos como mujeres. El desarrollo de una subcultura homoérotica urbana, por lo menos en la ciudad de México, será evidente después de la revolución hasta nuestros días, con circunstancias similares a las de Europa con respecto a la urbanidad y la moral.

La medicina moderna presentó argumentos anatómicos y sociológicos que contribuyeron en términos propiamente psiquiátricos, primero a la propuesta de un sujeto "uranista" (Ulrich, 1864), y después, del "homosexual" (Benkert, 1869), como enfermo con un "impulso sexual contrario" (Westphal 1870) o con "inversión del sentido genésico" (Charcot y Magnan, 1882), antecedente de la posterior teoría de las perversiones que explicaba la conducta homosexual y un tipo de sujeto social enfermo (Véase Vázquez García, 2001:149-150). De esta forma se hizo presente la imagen de sujeto homosexual en los relatos de la literatura médica, crónicas de sucesos y la sensibilidad pública.

En México, el discurso médico del siglo XIX tomo particular atención en controlar la prostitución y aplicar campañas de sanidad para mejorar las condiciones de salud de la población, 
justificando las redadas en las zonas rojas o de tolerancia en donde la presencia del encuentro sexual anónimo también se realizaba. No obstante, no existió en México una criminalización penal de los actos homoeróticos (Véase Gallego Montes, 2010:86-87, citado por Rodríguez Sánchez, 2018:118), pero sí en determinados periodos se registró un mayor número de detenciones, que respondían a proyectos políticos o a formas de evidenciar las acciones en favor del orden público. Lo constante fue la extorsión policiaca, un problema de corrupción, con falsas denuncias y acusaciones que continúan siendo comunes hasta el presente.

En la cárcel de Belem, y después de 1933 en la penitenciaría de Lecumberri, eran encarcelados aquellos hombres que no tenían los medios económicos para librarse de la detención, que por lo regular era una multa de no más de 20 pesos o la pena de no más de 20 días de cárcel, pues el verdadero delito era el de corrupción de menores y la razón de los arrestos era por escandalo o faltas a la moral pública. En Lecumberri, uno de los centros carcelarios más modernos del momento, existió un pabellón especial para confinar a los delincuentes sexuales, travestis y homosexuales detenidos por ejercer la prostitución o por escándalos en el espacio público, la crujía J, era la de delitos ambiguos, en ella estaban los "jotos", homosexuales orgullosos a pesar de su detención con poses insinuantes y sínicas, actitud irreverente y divertida que hasta hoy significa la práctica del “joteo” (Véase Rodríguez Sánchez, 2018).

\section{La heterosexualidad obligatoria}

Todos los cuerpos y las sexualidades permanecen vinculados a una heterosexualidad obligatoria institucionalizada por medio de arreglos legales, culturales y económicos en donde se subordina lo femenino y todo lo contrario a las prácticas disidentes de la norma heterosexual generan violencia y exclusión (Rich, 1993:227). El homosexual es útil para regular un orden y debe permanecer oculto y reprimido por el sentimiento homófobico, pero cuando el secreto se torna público, el peligro debe ser eliminado a fin de marcar la diferencia y recuperar la "identidad heterosexual coherente" (compárese Mason, 2001:3, citado por Gómez, 2007:75). Angela Harris (2000) señala que la violencia de género existe entre hombres, asociada a sentimientos de atracción y repulsión, dado que la identidad masculina es inestable. Las relaciones con otros hombres están marcadas además por condiciones desiguales como raza y clase social, determinando las posiciones o privilegios de la masculinidad, aun en las prácticas o identidades 
subordinadas, y se desarrollan formas alternativas para probar esa masculinidad (Véase Angela Harris, 2000:779-782, citado Gómez, 2007:77). Así, se explica el sentimiento homofóbico, un sentimiento por la pérdida de la masculinidad presente también entre los homosexuales, que se sienten atraídos y repelidos por el mismo objeto, lo que crea la necesidad de marcar formas de distinción (Véase Mason, 2001 y Harry, 1990).

Las practicas no hegemónicas de la sexualidad y las nuevas identidades se configuran a partir de la violencia o tensión que existen entre la prohibición de prácticas homosexuales, el concomitante deseo por lo prohibido, la violencia con que se normaliza tal deseo y sus efectos. Una realidad signada por la violencia, el dolor, la vergüenza, el miedo y la culpa, las cuales tomarán diversos ropajes metafórico-discursivos y posiciones particulares (Véase Páez Casadiegos, 2006). Las relaciones de sexo anónimo entre varones, vinculada al culto al cuerpo, al ocio y a un elevado capital económico y cultural, genera sus propios excluidos, los cuales no pueden acceder a los espacios de socialización sexual disponibles en las grandes ciudades tanto públicos como privados; y en esas zonas de encuentro, también se reproducen los principales factores de estratificación social, aunque traten de mantenerse en el anonimato (Mas Grau y Langarita, 2015:292).

Una contribución de las críticas feministas a la teoría psicoanalítica, en particular la desarrollada por Monique Wittig señala que la homosexualidad constituye la mayor prohibición de la sociedad moderna (Wittig, 1992:28). Como ha señalado Guillermo Nuñez Noriega (2007), el estigma homosexual se crea sobre la ausencia o menor hombría, ya sea en la demostración de ciertas capacidades subjetivas en el gusto o atracción por el pene o la penetración, o en el afeminamiento o el amaneramiento, “[...] que parece una renuncia o transgresión a un proyecto ideológico personal y social, pero naturalizado, de ser hombre" (Núñez Noriega, 2007:254), por ello la identidad homosexual se ha asociado a malestares relacionados con cuatro emociones: miedo, vergüenza, culpa y tristeza, que en conjunto se han definido como "soledad gay" (Lozano, 2016:33, citado por Laguna Maqueda, 2019:74). Los encuentros sexuales anónimos están claramente marcados por los criterios de la sexualidad hegemónica, una clara segregación sexual que hace que conductas o deseos se den en un contexto de hostilidad sexual marcada por normas y consensos (Langarita Adiego, 2013:313). 


\section{La reivindicación política}

Una referencia más próxima que implicó el cambio de esos discursos condenatorios se produjo desde la reivindicación social de las minorías sexuales a finales de los sesenta en Norteamérica, y después de 1969 en Europa, (Véase Pecheny, 2001). La concepción de la identidad gay inauguró una forma entender y experimentar las relaciones homosexuales (Véase Drucker, $2000 \mathrm{y}$, Balbuena y Ovalle, 2013), también hizo posible dar una mayor variedad de significados al sexo rápido, anónimo y sin compromiso.

Este nuevo posicionamiento de la identidad o "poder gay" se inscribe en la trayectoria de otros movimientos sociales de la década de los años setenta, y que han mantenido un debate crítico en torno a la heteronormatividad, como es el caso de los movimientos feministas, con su defensa de la libertad en la vida privada que incluía un conjunto de reivindicaciones que excedían esa dimensión, pues la revolución sexual era inseparable de la revolución social y política. (Felitti, 2006:49). En esos años surgió la moda unisex, se practicaba el sexo colectivo, los homosexuales y las lesbianas encontraron formas para expresar sus preferencias y ahora, se tenían el respaldo de la Asociación Americana de Psiquiatría quien reconoció la violencia en contra de esa orientación sexual, declarando en 1973 que la homosexualidad no era una enfermedad mental. Diez años después, en 1990, la Organización Mundial del al Salud retiró de la clasificación Internacional de Enfermedades a la homosexualidad; pero la persistencia de la idea de enfermedad o patología justificó la declaración por el mismo organismo internacional del año 2000, en la que se niega la eficacia científica de diversas terapias de conversión o cura de la homosexualidad.

La nueva configuración de la práctica sexual como identidades sexuales en México, como en otras partes del mundo, puede explicarse como un proceso de construcción identitaria entre los argumentos externos y locales con diferentes momentos importantes. Alejandro Brito (2010) diferencia tres etapas: En un inicio, con la salida al espacio público de 1978 a 1984, en donde se hablaba de homosexualidad y no de gais. Un segundo momento con la irrupción del VIH-SIDA, cuyos primeros casos se presentaron en México entre 1983 y 1984. El padecimiento permitió que la homosexualidad se trasladara de lo privado a lo público, como afirmó César González (2001), y una tercera etapa que se inicia en 1999 y que perdura hasta nuestros días. Esta última etapa se inició cuando la marcha del Orgullo LGBT (Lésbico, gai, bisexual, transgénero y transexual) de 
la ciudad de México llegaría al Zócalo por primera vez, lugar simbólico de las luchas sociales que marcó así una etapa de aceptación y búsqueda de plenos derechos (Véase Sánchez Domínguez, 2001:93-98).

Los estudios modernos en torno al sexo anónimo se han justificado también siguiendo los siguientes referentes. El primero consolidado desde el siglo XIX, partió del discurso médico, siendo central la categoría de perversión, la inmoralidad o la criminalidad. Langarita Adiego (2013), señala que el tema toma presencia fundamentalmente a partir de los trabajos de Laud Humphreys (1970), y posteriormente de Meredith R. Ponte (1974), y Richard R. Troiden (1974), quienes mantenían un discurso de criminalidad y desviación. El segundo, con la aparición del VIH-SIDA y la necesidad de prevención. En el caso de España, Langarita Adiego (2013), reseña las investigaciones de Oscar Guasch (1991), Paul Flowers, Graham Hart y Claire Marriott (1999), Jonathan D. Huber y Peggy J. Kleinplatz (2002), William Elwood, Kathryn Greene y Karen Carter (2003), Richard Tewksbury (2008), Fernando Villamil y María Isabel Jociles (2008; 2011) y Fernando Lores (2012). En un tercer referente, concebido gracias a las aportaciones de los estudios Lésbico-gais y las teorías deconstructivas, se puede tener en muchos casos la visión interior de las identidades y sus prácticas corporales, en este punto en particular podemos señalar las investigaciones en México de: Sánchez y López (2000), Núñez Noriega (1994), List Reyes (2003), Laguarda Ruíz (2011), Alberto Teutle y List (2015), Hernández Sancén (2016) y Sánchez Domínguez (2002).

Si bien el sexo anónimo es ejercido por hombres y mujeres en sus diferentes combinaciones, ha sido, y sigue siendo, particularmente atractivo entre los varones. Actualmente y de manera generalizada, esta práctica es conocida internacionalmente como cruising (Reece y Dodge, 2004). Pero en México, se le identifica bajo diferentes nombres como: "ligue", "putear", "vacilón" o "cotorreo", todas asociadas también a diferentes situaciones cotidianas o regionalismos; tienen en común ser prácticas silenciadas, permanecer invisibilizadas y ser casuales. Son muchos los atractivos emocionales del sexo anónimo como: el misterio, la libertad de sentir y hacer algo prohibido, la no implicación de sentimientos y compromisos, pero en la vida real las cosas no son tan casuales y en el caso de México, el sexo entre desconocidos se desarrolla de una manera más planificada, ritualizada y en un escenario facilitador. Por lo menos en la ciudad capital, las 
desigualdades económicas y sociales, el racismo, la violencia, la corrupción y la delincuencia, hacen de esta práctica foco de agresión, donde lo constante es el riesgo, y quizá también por ello su atractivo.

El sexo anónimo, desde la crítica al modelo interpretativo del sexo entre varones del que habla Núñez Noriega (2001), bajo el título de "modelo dominante de la comprensión del homoerotismo entre varones en México", es un fenómeno que aporta argumentos deconstructivos del binarismo que en México ha explicado el homoerotismo masculino como: una dualidad complementaria masculino-femenino, en la forma de "penetrador -penetrado" o "activo -pasivo". "hombre o joto". Se trata o de un modelo tradicional de explicación coherente con la heterosexualidad hegemónica, que permite entender las interacciones masculinas, pero que invisibiliza aspectos de esa realidad.

\section{Los lugares}

La ciudad es el lugar para la expresión homosexual, en muchos casos un refugio desde donde se configura su "otra familia" y se comparte el "estilo de vida gay"; un contexto al que se le ha sumado la cultura del consumo y en donde es mucho más frecuente el encuentro sexual anónimo. Las zonas, lugares o barrios sexuales en donde es posible el sexo casual, siempre han coexistido con el resto de las funciones urbanas desde tiempos inmemoriales. En la ciudad de México existe una gran cantidad de posibles lugares de encuentro homoerótico, algunas zonas conocidas de comercio sexual, junto con lugares de encuentro y diversión para homosexuales y otras identificadas como "no lugares", replanteando lo propuesto por Marc Augé (1992), dado que, muchos de ellos son ocupados temporalmente y responden a otros usos sociales que al ser silenciados son intersticios inexistentes, solo fantasmas de lugares para los actos sexuales del anonimato.

Para su presentación, estos sitios los hemos dividido en dos grandes grupos: los espacios abiertos (áreas al exterior) y los cerrados (lugares al interior); que a su vez se subdividen en libres (espacios públicos o de libre acceso), y comerciales (que están enfocados a un tipo de servicio público o con acceso específico). Cada uno de ellos conllevan diferentes posibilidades y riesgos, que condicionan los encuentros. 


\section{Espacios abiertos y libres}

La calle es el espacio público por antonomasia, es donde las personas expresan su identidad y la construyen, se solidarizan, crean redes sociales naturales y entran en contacto directo con desconocidos (Salazar Barrón, 2016:103). En México los sitios más tradicionales de encuentro social son los parques centrales, las alamedas o las plazas; lugares en los que en el pasado podían encontrase miembros de todas las clases sociales, contexto para el galanteo y la vida licenciosa. Dentro de la ciudad de México, destaca hasta hoy la Alameda Central, que desde principios del siglo XX es considerada un lugar de encuentro homoerótico, pero todos los parques o plazas públicas pueden ser utilizados para el encuentro.

Desde la caída de la tarde hasta las primeras horas de la madrugada, la Alameda Central es el refugio obligado de pederastas y 'buscones' que hacen imposible transitar por la noche por dicha parte, pues las insinuaciones, los gritos, las señas, los ademanes y los gestos obscenos de los afeminados pululantes resultaban intolerables para las personas decentes (Jaime Luna. Los homosexuales y las tribadas. Detectives, 25 oct. 1937:30, citado por Rodríguez Sánchez, 2018:156).

Entre los jóvenes universitarios es famoso desde la década de los ochenta el llamado "Camino verde" un espacio libre dentro de la ciudad universitaria en el que es posible tener encuentros sexuales, también dentro de las instalaciones de la universidad nacional están los circuitos del jardín botánico en donde el encuentro puede darse a pie, en bicicleta, en moto o en automóvil, dependiendo del horario. Otros parques en los que es frecuente el encuentro homoerótico son el Parque Hundido o el Bosque de Tlalpan (Véase Sánchez Domínguez, 2002).

Aunque por sus cualidades de alumbrado o tránsito algunas calles son particularmente propicias, la experiencia del "ambiente" señala a la esquina de Aguascalientes e Insurgentes, como la "Esquina Mágica", un lugar donde muy probablemente se lograría un "jale" o "levantón”, pero desde la década de los sesenta, la zona con mayor potencial para el ligue callejero sigue siendo la "Zona Rosa":

Era realmente preciosa. Mucha moda, mucha beautiful people. Todavía conservaba buena parte de sus impresionantes casonas europeas de principios de siglo. Estaba llena de aparadores deslumbrantes y de turistas rubicundos y sonrientes, lo que le daba cierto resplandecer diurno. Galerías de arte, boutiques, mexican curious, antigüedades; hoteles, centros nocturnos y restoranes de lujo; agencias turísticas, tiendas de discos importados y hasta de filatelia; academias de idiomas 
y de modelaje, y se podía caminar con tranquilidad (todavía no llegaba el metro, ni con él la muchedumbre de muchachos de barrios pobres). Era uno de los escasos sitios donde cualquiera se permitía andar, impunemente, vestido de hippie, o con ultra minifalda y hot pants, o con atildada melena de Beatle y pantalones ajustados, acinturados, destacando las nalgas y el paquete, y de colores extravagantes, lo que provocaba insultos, golpes y aun detención policiaca como en el resto de la ciudad (Blanco, 1997:78, citado por Laguarda, 2010:156).

Dentro de este grupo de lugares están también las playas, en donde es posible un encuentro casual, aunque fuera de la ciudad. Destaca la llamada "Playa del Amor" en Zipolite, Oaxaca, playa nudista visitada regularmente por turistas nacionales y extranjeros en la que son posibles los encuentros sexuales individuales o colectivos. Durante el día, a partir de buscar un lugar más privado entre los riscos de la playa, y durante la tarde y noche, bajo una relativa invisibilidad que autoriza la interacción con la proximidad y la participación colectiva. 


\section{Espacios públicos más privados}

A este grupo corresponden las estructuras que en algunos lugares acompañan a los sitios abiertos y públicos, con un lugar primordial: los servicios como baños públicos, mismos que en algunos lugares han construido toda una tradición o mito popular que hace realidad los lugares como sitios de encuentro sexual. Los baños públicos más accesibles para los jóvenes con fines homoeróticos tienen que ver con el uso de instalaciones deportivas y áreas comunes como vestidores y regaderas. También están los baños de escuelas o facultades, donde por su ubicación son propicios, entre los jóvenes de la ciudad de México, famosos eran los baños del Anexo de Ingeniería y del Posgrado en Arquitectura, en donde los estudiantes se encargaban de hacerlos adecuados, perforando las paredes de los privados. La distribución de los espacios hace atractivos los lugares, la posibilidad de ver, de ocultarse o estar cerca. Por ejemplo, los espejos del baño del Palacio de Bellas Artes, con mingitorios no adosados al muro, o la distribución por corredores separados en las terminales de autobuses, como en los baños de uso gratuito de la terminal de San Lázaro.

\section{Los espacios comerciales abiertos}

En este grupo están los parques temáticos, balnearios, campamentos, hoteles y fincas. Son sitios a los que se accede mediante el pago de una cuota o membresía y que se convierten también en alternativa para el encuentro y placer homoerótico. Destacan por la cercanía con la ciudad de México la oferta de balnearios de los estados de: Morelos, México e Hidalgo. Los más visitados en la década de los ochenta eran el Centro Vacacional Oaxtepec, las Estacas, la ex hacienda de Temixco y más recientemente el Rollo y El Tepe. Un centro recreativo como estos, pero enfocado a la población gay se abrió en Tepoztlán en Morelos, un spa que funciona los fines de semana ofreciendo además de masajes, cuarto oscuro y restauración, espacios de descanso, albercas y jacuzzis.

\section{Espacios comerciales apropiados}

Corresponde a los muchos sitios de moda o lugares prestigiados como librerías, restaurantes, centros de espectáculos teatros cines, etc., los cuales no fueron hechos para propiciar los encuentros sexuales pero que la clientela que los visita los utiliza con ese fin. 
Los Sanborns, en general, eran buen lugar para ligar, desde la mañana hasta la noche, a toda hora. Estaba el Sanborns de Aguascalientes, que era el más famoso por su concurrencia gay. Por supuesto que el de San Antonio y el de San Ángel, claro; y el del Ángel. Muchas parejas rompieron al equivocarse en el lugar de la cita: "nos vemos en el Sanborns San Ángel" y el otro entendía que era el "del Ángel", ambos se quedaban plantados y, entonces, el amor acababa. Era un lugar para conocer jóvenes universitarios o profesionistas jóvenes. En el baño, en las escaleras de la entrada, en las revistas, echabas una mirada, veías qué había. Ahora, siempre tenías que estarte cuidando de los empleados y de los judiciales que te quitaban todo lo que traías "por puto", así te decían. Y te amenazaban, ya sabes: "le vamos a decir a tu esposa o a tu familia". Una vez me tocó uno y le dije: "¿cuál esposa, cuál familia?" Pero, en general, en los Sanborns se hacía de todo, adentro de los baños. Era impresionante. Se armaban orgías, verdaderas orgías, y un alma caritativa siempre era el que daba el pitazo para que los empleados no vieran el aquelarre, qué cosa (Testimonio de Ignacio reproducido por Laguarda, 2010:167).

Así, los homosexuales se apropiaron de la cadena Samborns desde los años sesenta y algunos otros establecimientos como gimnasios, cafeterías y cervecerías. La cervecería "Elba" en la calle de Uruguay, la "Cuate", de la calle Lucas Alamán y la "Primera Lucha", estuvieron de moda hasta mediados de la década de los años cuarenta y en los ochenta fueron famosas las cervecerías: "el Oasis" y "el Viena", "Bar La Lili" o "La tortuga" junto con nuevas ofertas como "La purísima”, "La Puerca", “Cabaretito”, "Marrakech”, "Pecado” y "Vaqueros Bar".

Desde las primeras décadas del siglo XX diversiones como el teatro y el cine fueron un lugar favorable para el erotismo y sexo entre varones, se conoce el Montecarlo, teatro de ínfima categoría ubicado en la Tercera demarcación de Policía, en cuya oscuridad pululaban los tocamientos entre hombres, como también sucedía en el cine Briseño y en el Politeama (Véase Rodríguez Sánchez, 2018:133). En los años sesenta, fue famoso el cine "Roble”, sobre el paseo de la Reforma, un cine al que asistía mucha gente de "ambiente" y el ligue era constante, lo mismo que en los cines que ofrecían películas sexuales como el cine "Gloria” o el "Teresa".

Los baños públicos, vapores y saunas fueron siempre un lugar de la intimidad masculina y siempre han sido utilizados para el sexo casual, a pesar de la existencia de letreros exteriores que señalaban la prohibición de acceso a homosexuales y amanerados, en la práctica la mayoría de los baños ofrece masajes que pueden incluir servicios sexuales, a la par de los contactos casuales o encuentros colectivos. En la ciudad de México famosos son los baños: Ecuador, San Juan, Rocío, Finisterre, Xochimilco, San Cristóbal, Acapulco y muchos más. 
RELIES: Revista del Laboratorio Iberoamericano para el Estudio Sociohistórico de las Sexualidades. Número 3 (2020) 


\section{Los espacios especificos}

Estos lugares comerciales corresponden a la oferta explicita para un público sexo diverso, amistosos a ellos o declaradamente sitios gais. Los primeros de estos negocios de la ciudad de México fueron bares que se crearon en los años setenta, los cuales fueron mejorando su servicio y ofreciendo un lugar para cada gusto, la mayoría lugares cerrados poco iluminados con diferentes ambientes. Para la década de los años setenta ya existía un buen número de ellos. Quienes se reconocían como homosexuales y querían expresar su identidad tenían que asistir a los bares, espacios de libertad que fueron gradualmente ofreciendo más facilidades para el contacto sexual como el "Bar 9", "El Don", "Le Barón”, "El Topo", "El Deval”, "El Penhouse”, "Famoso 41”, "El Taller", "El Tom's”, “El Living", "El Marrakech", “El 14”, "El Anyway”. "Butterflies y "Espartacus".

Otros servicios como librerías, cafeterías y restaurantes son más discretos. El espacio de lectura, los baños o las barras son propicios para el contacto visual y quizá algún acercamiento discreto o "arrimón", aunque es poco frecuente el contacto sexual también puede llevarse a cabo, en especial en espacios más privados como los sanitarios.

\section{Cabinas, Clubes sexuales y saunas}

Las tiendas de productos sexuales que cuentan con servicios de cabinas en las que se pueden ver videos o películas pornográficas son un punto para el sexo casual. Algunos usuarios dejan abiertas las puertas, invitando a los curiosos a interactuar. Pero los más acondicionados son los clubes específicos para el encuentro sexual que según su tipo, tienen áreas especiales de gimnasio, sauna, vapor, regaderas, sala de espuma, alberca, salón sadomasoquista, privados, camastros colectivos para orgias, paredes perforadas, salones para fumadores, salas de videos y espacios laberinticos que, por supuesto cuentan con guardarropa, haciendo costumbre el estar semidesnudo o desnudo al interior. Famosos en la ciudad de México son "La Casita", "La Toalla" y "El Sodome".

\section{Los transportes}

Otro de los sitios públicos fundamentales para el encuentro homoerótico y sexual son los transportes, autobuses, taxis colectivos, metro o tren, en especial en los horarios de mayor 
demanda o en los horarios con menor número de pasajeros. El más emblemático es el metro, pero dentro de las fantasías y relatos de encuentros sexuales, se encuentra todo tipo de transportes incluidos los aviones o los barcos. El "metreo" acción de buscar encuentros sexuales en este medio de transporte, también es una práctica que se desarrolla en casi todas las líneas del metro de la ciudad de México, al lado de la prostitución, el comercio informal y el narcomenudeo.

Algunas líneas son más propicias por la cantidad de pasajeros, pero no en todo momento, pues existen por horarios, circuitos para la búsqueda de la experiencia sexual y ciertas referencias que hacen exitosa esa búsqueda entre los participantes. Por convención se ha identificado el último vagón de cada línea como propicio para el encuentro homosexual, llamado también el "putivagón” (Véase Hernández Sancén, 2017). El metro cuenta con 12 líneas o rutas que conectan diversos puntos geográficos de la ciudad en una amplia red de túneles en donde los transbordos, accesos, corredores y vagones se convierten en oportunidades de encuentro entre desconocidos.

\section{Sitios Virtuales}

Una nueva categoría de espacio que ha adquirido mayor popularidad es el virtual, a partir de Apps, páginas de encuentro o chats, que permiten usar las redes sociales y los dispositivos digitales para saber si existen en el entorno parejas sexuales potenciales o compatibles, además de las posibilidades de cibersexo, a través de la web-cam, también habitual entre personas que no se conocen. Estos recursos, más utilizados por los jóvenes, proporcionan una falsa confianza o seguridad, además de ser aún más excitante el encuentro, que será fortuito, rápido y clandestino entre dos amantes improvisados en los lugares más inesperados.

\section{Psicogénesis en prácticas}

En el juego del placer homoerótico el género es espacio y práctica: En los lugares públicos de la ciudad el género se actúa, legitima o se niega, se produce mediante el cuerpo, sus movimientos y sus deseos. Jordi Mas Grau y Langarita Adiego (2015) subrayan que para lograr mantener relaciones sexuales en estos espacios es más importante acatar las normas implícitas que emplear una buena estrategia de flirteo. El cuerpo, y en especial el pene, es aquí el "artefacto simbólico supremo", se muestran los genitales sin pudor y se ocultan los datos personales (Véase Mas Grau, 
y Langarita Adiego 2015:191) En el contexto de los diferentes lugares posibles permanecen como principios elementos ritualizados y simbólicos que permiten entender y participar sin mediar palabra, por lo que son fundamentales el anonimato y el silencio.

\section{El anonimato}

A pesar de las oportunidades de experimentación que brinda la ciudad, las prácticas sexuales no dejan de ser objeto de críticas, la ciudad misma es considerada como el lugar que acoge las mayores perversiones, responsable de la pérdida de valores y tradiciones, donde se engendra la depravación moral (Véase Langarita Adiego, 2014b). En ese contexto existen diferentes discursos que sancionan la sexualidad anónima, marginando las practicas homoeróticas a espacios de silencio y clandestinidad.

Bajo la mirada sanitaria y la de una salud sexual, se advierte de las enfermedades de transmisión sexual y las perversiones. Las advertencias sobre la conducta delictiva en torno a los lugares de encuentros hacen indispensables las advertencias y los consejos de seguridad para no acudir sólo la primera vez, estar alerta y detectar posibles peligros, buscar lugares seguros y salidas rápidas en caso necesario. La mirada social identifica a los participantes por su apariencia e intenciones, sancionando que se superen los límites de permisibilidad de los lugares, identificados como despersonalizados y peligrosos, como si en ellos se llevase a cabo un sexo no social, promiscuidad o excesos que dañan la imagen personal y la del colectivo homosexual. Estos discursos negativos se contraponen a los relatos literarios que hacen referencia explícita a la práctica del sexo anónimo y describen las bondades de los encuentros entre hombres haciendo fantasear al lector y trasladándole los sentimientos, deseos y sensaciones íntimas que se pueden vivir en este tipo de lugares, fomentando un imaginario.

Dentro de estos significados subjetivos sobrevive el pensamiento homofóbico. Langarita Adiego (2014b) resumen tres de los principios de dicho pensamiento. El primero la condena a las relaciones sexuales entre personas del mismo sexo, una relación no reproductiva y contraria a la heteronormatividad. En segundo lugar, la monogamia, como la forma legítima de relación que se opone a la infidelidad o a las parejas casuales, y por ello es necesario el ocultamiento. Por último, el valor del amor romántico como principio para la sexualidad y sus lugares adecuados. Este 
hecho hace difícil que los propios participantes escapen del reconocimiento de lo abyecto y articulen discursos positivos de su experiencia. Sus propias historias son polimorfas, cada sujeto encuentra un sentido distinto a lo que allí sucede. Son al mismo tiempo historias de control y liberación, de supervivencia, de deseo y de perversión, que confunden lo sucedido con lo imaginado. Son también narrativas de la mentira y del desprecio, segregación, discriminación y hostilidad sexual; un anonimato que se torna reafirmación de los valores de la masculinidad hegemónica.

\section{El silencio}

Uno de los principios de esta complicidad masculina es el de silencio, conjuntando el anonimato bajo la lógica de la heterosexualidad hegemónica, el significado estigmatizante de la homosexualidad y el atractivo de la transgresión. Langarita Adiego (2014b), señala que las prácticas del sexo anónimo están claramente ritualizadas haciendo que se produzca una atención y emoción entre los participantes que proporcionan una realidad temporalmente compartida, para ello cuentan con un conjunto de reglas propias que determinan cuando se inicia y acaba la interacción, dimensión compartida por un grupo que la interpreta y la respeta.

Víctor Turner (1980:35), clasifica los símbolos en dominantes e instrumentales. En este caso, el paradigma simbólico dominante es la heterosexualidad, bajo la forma de homofobia; y los símbolos instrumentales, son aquellos que tienen un fin específico dentro del ritual. Bajo el concepto de Pierre Bourdieu de "habitus" (1984), el cuerpo es reconocido y en él se materializan las relaciones sociales de poder, creando instrumentalidad entre las conductas que lo han marcado y las que desea. Son en principio los símbolos inherentes a su corporalidad y que el sujeto difícilmente puede manipular, pero que son un elemento fundamental para la interacción como: la edad, la etnia, la capacidad muscular o flacidez, la obesidad o delgadez, el olor o el vello corporal, atributos que son valorados desde la masculinidad hegemónica. Un segundo nivel instrumental son los símbolos del cuerpo manipulables, estos son utilizados conscientemente por los participantes. Un sistema de comunicación corporal en el que se destaca: el mantenimiento de la mirada; la demostración genital y los desplazamientos. 
La mirada es un acto de comunicación que sirve para reconocer el interés y disposición entre los participantes, una estrategia que requiere de la complicidad (Tewksbury 1996). La mayor parte de los hombres recurren a la estimulación genital para demostrar su deseo y necesidad sexual, propiciando una interacción que se negocia paso a paso.

Nos colocamos enfrente y, sin mediar palabra, nos pusimos a masturbarnos mutuamente. Después me propuso que le mamase la polla, a lo que le contesté que no. Le propuse que lo hiciese él, pero tampoco quiso. Me ha insinuó que quería follarme y me negué. 'Entonces te haré correr de gusto', me advirtió. Me masturbo un rato hasta que me corrí. Se sacudió el semen de la mano y se limpió restregando su mano en la pared, yo me subí los pantalones y me marché de la zona de cruising" (Extraído de mi diario de campo, Langarita Adiego, 2014b:11).

El silencio potencializa el lenguaje corporal en la negociación, más que un encuentro fortuito es una estrategia de contacto que satisface un deseo personal, una imagen o una sensación conocida y repetida, bajo la novedad de una búsqueda exitosa. El silencio protege la identidad social y el cuerpo ofrece el placer buscado. Es sexo puro, duro y sin compromisos. Dentro del silencio se reafirma lo marginal de las relaciones y se cosifica su significado.

Sin presiones se autoriza a marcharse sin mediar palabra y retomar de nuevo la búsqueda en favor de otras parejas sexuales sin la necesidad de atender a la satisfacción de la pareja previa. Algunos participantes pueden continuar una dinámica sexual en la que no se sienten cómodos, pero prefieren atenerse a la situación por respeto a la norma social sin plantear los problemas que se generan en la interacción, es por esa razón que resulta complicado en ocasiones el uso del condón (Véase Elwood, Greene, Carter, 2003:290).

Entre varones la mirada es ante todo un reto, una afrenta, que debe convertirse en erótica, pero sin feminizarse, sin perder valor masculino. La mirada denota atracción, interés, vinculo que se puede interpretar como agresión o placer, este primer indicio debe aclarase con las actitudes del cuerpo y las acciones, movimientos, forma de aproximación o simple imitación para reforzar que existe una comunicación. El ritual de aproximación, de ceder o retirarse, es el juego que da sentido al contacto, pues lo importante es el control de uno mismo y su placer, poco importa el otro, es la imagen propia la que se cuida y con la que se compite.

Miradas, genitales mostrados, caricias insinuadas y la proximidad, dan pie al intercambio cuerpo a cuerpo, tras la valoración del otro, su aroma, su altura, su musculatura, el tamaño del miembro, 
o cualquier otra fijación corporal complementa el paso a la sexualidad, que puede ser masturbación mutua, felación o penetración. El cuerpo esta mapeado y jeraquizado en donde el órgano genital, como instrumento de intercambio, se convierte en un elemento constituyente de persona (Langarita Adiego, 2013:328). Sentimientos y deseos sexuales son una cosa, mientras que la identificación, posición social o identidad, es otra (Weeks, 1998: 213-220, Citado por De Simone, 2018:5).

La búsqueda de placer se traduce en minutos o quizá horas de circulación de cuerpos recorridos y palpados, que solo se satisfacen de momento con la participación colectiva, la llamada "rebatinga", en donde entre más hombres más libertad para tocar y ser tocados, sin límites ni posibilidad de huir. El rechazo es también codificado, desde quitar o impedir que una mano toque el cuerpo, alejarse, con advertencias verbales, dar un empujón o golpe y abandonar rápidamente el lugar, una teatralidad de la agresividad masculina. El encuentro sexual más común es el oral, lo que establece claramente las distinciones de sujetos y el resguardo de su masculinidad, considerando la pasividad como cualidad femenina no deseable, por lo que la penetración no es una simple forma de relación o práctica, se acepta y se goza desde otros significados corporales o identitarios. Aunque en este juego de poder parece que no hay responsabilidad con respecto a las relaciones o placeres de otros, el proceso de selección y valoración a partir de las cualidades del cuerpo genera rechazo y discriminación, o por lo menos insatisfacción, desvalorización y frustración entre quienes no cuentan con las cualidades corporales deseadas: la juventud, genitales grandes, el aprendizaje de los códigos adecuados o la conciencia del significado de las prácticas de dichos lugares. No obstante, existe la necesidad del placer y cacería de cuerpos, donde se crean y reproducen códigos de valorización corporal, naturalizando entornos de discriminación, pero experimentando en los encuentros nuevas dimensiones de la complicidad y lo erótico.

\section{Explicaciones y placeres}

Las razones por las que los hombres recurren a estos espacios de homosociabilidad son muy variadas, pasan por la psique individual, teniendo en algunos casos explicaciones prácticas, como satisfacer sin compromisos una necesidad corporal hasta aquellas que no logra ser claras para los 
propios participantes. Lo mismo sucede con las prácticas sexuales, un goce individual con niveles o escala de satisfacción marcados por sus imaginarios.

Es difícil tener un testimonio de quienes participan en estos encuentros resguardados por el silencio, a pesar de poder ser testigos de sus prácticas; es por ello que se retomaran en este apartado algunos testimonios publicados en el texto de Teutle y List (2016). Estos autores relatan las experiencias de los usuarios de baños y saunas de la ciudad de Puebla, y nos muestran la interpretación del sentir de quienes acuden a estos lugares para tener sexo anónimo. En opinión de los autores, la asistencia a esos espacios responde a la satisfacción sexual y la búsqueda de placer, en un ambiente que no crea vínculos ni compromisos (Teutle y List, 2016:18). Estas prácticas que en muchos casos son producto de la actuación de papeles o roles estratégicos para generar una forma de relación entre los sujetos, dependiendo como ellos mismos y otros las interpreten y de la subversión que ellos mismos son capaces de reconocer. Un testimonio señaló:

Todo depende de que busques aquí en el baño, Si tú buscas muy "Chacales" así muy macho, pues lo que tienes que hacer es afeminarte, eso le llama la atención a los "mayates" a los "machotes". Sí tú buscas una loca, al contrario, debes de comportarte muy macho, no "jotear", ni nada. Pero locas todas las de aquí son, sólo se valen de la imagen (La Concha, 43 años, soltero, reproducido Teutle y List, 2016:150).

Las construcciones de género, las identidades y la vida social, aunque silenciadas, reproducen el orden heteronormativo y son requisito para obtener un contacto sexual satisfactorio, señalando que no todos son iguales, como lo comenta un padre de familia:

Yo soy chofer y por lo regular vengo a Puebla cinco veces al mes. La primera vez que topé con los baños éstos, fue porque di de "chiripa" y me empezaron a seguir algunos de los "chavos" que andan "cotorreando" ahí dentro. Yo no quería hacer nada, pero al final me convencí, estaba lejos de mi casa, de mi esposa, de mis hijos, no habría posibilidad de que ellos me encontraran o supieran, por eso de vez en cuando vengo a calmarme la "calentura" (Eder, 42 años, casado, reproducido por Teutle y List, 2016:51).

Los hombres que acuden pueden tener una experiencia placentera, pero no todos tienen una explicación del porqué lo hacen, el placer, en su lógica o moral, implica remordimiento y vergüenza, que explica lo que señaló Jorge: 
Es que a mí me trae el diablo, esta es la respuesta más lógica, la única forma es que el diablo me lleva a los baños y yo lucho contra eso y no puedo (Jorge 42 años, divorciado, reproducido por Teutle y List, 2016:85).

No es extraño que, ante la posibilidad de tener una satisfacción sexual, los participantes olviden que son observados y evaluados, expresen sonidos de placer, movimiento o actitudes que al terminar crean cierta vergüenza, además de la propia homofobia o problema de auto aceptación. Es frecuente que después de tener sexo, oral o penetración, los participantes salgan rápidamente del lugar ya sea para asearse o retirarse sin ser reconocidos, algunos cubriéndose genitales o el rostro, una posición contraria a la previa actitud propiciatoria del contacto.

La actitud retraída y cautelosa también es asumida por aquellos que se asumen como hombres heterosexuales, pero acuden para tener un placer gratificante como el sexo oral. Tratan en todo momento de controlar la interacción, de no dejarse besar, no dejar que toquen sus glúteos o que pierdan el control de la situación. En cuanto logran el orgasmo abandonan a la pareja o grupo de hombres que los ha satisfecho. No hay que olvidar que en las explicaciones vertidas se dan a partir de las propias trayectorias de vida, haciendo casi imposible una generalización, pero su naturalidad de sentido y lógica nos hace descubrir el poder de lo normativo. Actitudes diferentes toman los hombres que se asumen como homosexuales o gais, que buscan a otros valorados como más hombres, con mejores cuerpos, penes más grandes, agresivos y dominantes, así lo señala un testimonio:

A algunos hombres les ponen atención tal vez porque son "caras nuevas", como carne fresca y sobre los cuales acontecen, otra vez la lluvia de miradas sobre los cuerpos, sobre sus piernas, brazos, nalgas, rostro y, sobre todo, en su pene [...] (Toño, 25 años, soltero, reproducido por Teutle y List, 2016:78).

Lo complejo de las relaciones y significados, pasan por lo moral y por las fantasías de la identidad, los cuerpos masculinos, como señaló Oscar Emilio Laguna (2019) son evaluados conforme a los estándares homofóbicos tradicionales, en cuanto a su proximidad o alejamiento del modelo hegemónico, fundamental en el rol sexual, ser activo o pasivo, teniendo mayor prestigio quien tiene un rol activo que se distancia de las mujeres, noción presente en la homofobia interiorizada. Quizá por ello una de las fantasías habituales de algunos gais es tener 
relaciones sexuales, con hombres heterosexuales y preferentemente casados (Laguna Maqueda, 2019:78).

Con la caracterización complementaria sobre la identidad gay lo que se dibuja como sistema sexual mexicano es en realidad una especie de sistema homoerótico dual: uno de origen hispano, estructurado alrededor de la dicotomía activo-pasivo y otro de origen noreuropeo y norteamericano, articulado en función de la condición intercambiable de los papeles eróticos y de la noción gai" (Nuñez Noriega, 2007:281).

Hombres con cuerpos deseados, son los trabajados en gimnasios, los que acuden a ser vistos y encontrar a otros como ellos, con alto valor sexual desde la oferta de cuerpos, con los que se goza y se compite en capacidad o desempeño, un placer acotado a la duración o la cantidad de contactos o parejas, en este caso no importan las categorías de pasivo y activo, pues su calidad de hombre y su masculinidad incuestionable, pasa a adquirir otro valor.

Un modelo de género que es reconfigurado en la dinámica de las prácticas, done la penetración puede ser también una forma de placer de control y selección de lo masculino, como lo señaló un joven de buen cuerpo que es asistente frecuente a los clubes sexuales. Después de pasar toda una noche con varias parejas, señaló que no tuvo un buen día, pues sólo se "echo a 10 hombres", pues suele tener mejores días, es decir un número mayor de relaciones. Señalando que se cuida y que no se trata de cualquier hombre, son masculinos con excelente material, penes grandes, duros y que aguanten, con orgullo señala que: "hay que saber escoger hombre”. Lejos de sentirse usado al ser pasivo, es él quien los usa y controla, es quien tiene el poder, escoge y exprime a quien le gusta, una cacería y un trofeo que se traduce en el placer de haber tenido un buen resultado.

Sí se tiene una masculinidad viril, se tiene una posición social privilegiada (Teutle y List, 2016:149), una lógica social que está detrás de la aceptación y ordenamiento de la jerarquía de la sexualidad moderna (Rubin, 1984). Es quizá por ello, que quienes han adquirido una identidad "aceptable como gais" a partir de la política del orgullo, asuman una postura intolerante para con el sexo anónimo, privilegiando asumir la identidad sexual pública y la vida en pareja como adecuada, otros espacios en donde la vida gay ha ganado terreno:

Que la sociedad identifique a los gais con tíos que van a lugares apartados a darse por el culo con unos y con otros sin preguntarse ni siquiera el nombre, ¿crees que no perjudica nuestra imagen de cara a la sociedad? ¿Crees precisamente que eso no le da argumentos a la extrema derecha? Mi 
juicio de valor no es nada subjetivo. De hecho, es muy objetivo. Cada uno es libre de hacer con su cuerpo lo que quiera, pero aquí nadie está exento de crítica. No os pongáis hipersensibles, porque no sois intocables, $;$ eh! Lo creo personalmente, y siento mucho al que le ofenda, que esta práctica, que yo no comparto, no va asociada inherentemente a la homosexualidad, y yo no tengo porqué defenderla. Esto no tiene nada que ver con la homofobia. Llamadle "cruisingfobia"' (comentario de un lector en el foro de la página web de noticias LGTB http://www.dosmanzanas.com).

Esta opinión refiere de nuevo el fantasma de la promiscuidad y lo perverso. El perverso no es el que gusta del mismo sexo, sino el que no lo asume como gay, el que tiene una doble vida como bisexual, el que recurre al sexo simplemente por placer, un discurso de nuevo disciplinar.

[...] para que el modelo gay tuviera un hueco en el cajón de la organización sexual occidental ha necesitado distanciarse de las putas, de las locas, los y las transexuales, etc. Y demostrar que, de alguna manera, la homosexualidad no tenía por qué estar reñida con el modelo de masculinidad occidental, ni con la pareja, ni con la familia y ni mucho menos con el capitalismo. Prueba de ello es el crecimiento de un comercio gay, que se presenta como exclusivo para aquellos homosexuales que se pueden subir al tren del gran lujo. (Langarita Adiego, 2013a:337).

Lo cierto es que las prácticas de sexo anónimo entre hombres requieren y reproducen el deseo desde la hegemonía heterosexual y lo fálico, transgreden el ideal de pareja, el campo del deseo, del prestigio y del poder. Silenciando experiencias intimas, emociones e interacciones desgenitalizadas que siguen configurando actos transgresores del orden y formas diferentes de homoerotismo masculino, pues en esas experiencias se resignifican una y otra vez los encuentros y las prácticas.

\section{Comentario final}

Desde el siglo XIX se consolida la institucionalización de la heterosexualidad bajo los imperativos del género que también reconoce a un sujeto como homosexual, dando sentido a un patriarcado moralizante donde el deseo erótico debe ser desplegado bajo estrictos consensos sociales de lo privado. A pesar de las libertades sexuales modernas, la homosexualidad sigue siendo una sexualidad estigmatizada, el encuentro sexual anónimo no es un juego silencioso que hace uso del cuerpo para rebasar las fronteras impuestas del control sexual, es estrategia que responde a significados preexistentes como alternativas. 
El placer del sexo sin ataduras, libre y sin compromiso, sólo se logra asumiendo códigos, adecuándose a lugares apropiados o permitidos, respetando acuerdos silenciosos y adecuando el placer a cada sujeto. El homoerotismo se construye y reconstruye con discursos y prácticas, como un juego de poderes y resistencias. Al centrar su interés en lo fálico, se convierte en reafirmación de lo heteronormativo, pero en su dinámica también se construyen prácticas, que pueden ser más frecuentes y diversas, como espacios que no tengan que ver con un modelo de dominación / sumisión o actividad/pasividad y que hacen necesario entender su significado desde la complejidad que encierran. 


\section{Referencias bibliográficas}

Augé, M. (1992). Los no lugares. Espacios del anonimato. Una antropología de la Sobre modernidad. Barcelona: Gedisa Editorial.

Balbuena Bello, R. y Ovalle Marroquín, L. P. (2013). Organización política gay y espacio público. Trace 63, Junio 2013:50-59.

Blanco, J. J. (1997). Ojos que da pánico soñar. Función de media noche. Ensayos de literatura cotidiana, México: Era.

Bourdieu, P. (1984). Distinction: A Social Critique of the Judgement of Taste. Cambridge Mass: Harvard University Press.

Cardín, A. (1989). Guerreros, chamanes y travestis. Indicios de homosexualidad entre los exóticos. España: Tusquets Editores.

De Simone, R. L. (2018). Deseos urbanos: género, erotismo y consumo en la ciudad contemporánea. Planeo 26, febrero 2016:1-16.

Drucker, P. (2000). Different Rainbows. Prowler Group Ltd. - Londres: Millivres.

Elías, N. (1999). Sociología fundamental. Barcelona: Gedisa.

Elwood, W. N.; Greene, K.; Carter, K. K. (2003). Getlemen don't speak: communication norms and condom use in bathhouses. Journal of Applied Communication Research, 31 (4):277-297.

Felitti, K. A. (2006). En defensa de la libertad sexual: discursos y acciones de feministas y homosexuales en los '70. Temas de mujeres 2, 2006:47-69.

Flowers, P.; Hart, G.; Marriott, C. (1999). Constructing sexual health. Gay men and "risk" in the context of a public sex environment. Journal of health Psychology, 4 (4):483-495.

Foucault, M. (1986). Historia de la sexualidad, México: Siglo XXI Editores.

Gallego Montes, G. (2010). Demografía de lo otro. Biografias sexuales y trayectorias de emparejamiento entre varones en la Ciudad de México. México: El Colegio de México.

Gómez, M. (2007). Violencia Homofobia y psicoanálisis. Revista de Estudios Sociales 28, diciembre de 2007:72-85.

González Oquendo, L. J. (2014). La investigación sociológica figuracional de Norbert Elías: elementos conceptuales y metodológicos. Prácticas de oficio. Investigación y reflexión en Ciencias Sociales 14, diciembre de 2014:70-86.

González, C., (2001). La identidad gay: una identidad en tensión. Una forma para comprender el mundo de los homosexuales. Desacatos 6, primavera-verano 2001:97-110.

Guasch, O. (1991). La sociedad rosa. Barcelona: Anagrama.

Harris, A. P. (2000). Gender, Violence, Race, and Criminal Justice. Stanford Law Review, 52:777-807.

Harry, J. (1990). Conceptualizing Anti-Gay Violence. Journal of Interpersonal Violence, $5(3)$.

Herdt, G. (1997). Same sex, different cultures. United States: Westview Press.

Hernández Sancén, J. O. (2017). El "putivagón" y el "metreo" en la ciudad de México: lo material, lo social y lo imaginario, Seminário Internacional Fazendo Gênero 11 \& 13th Women's Worlds Congress (Anais Eletrônicos), Florianópolis, Brasil: UFSC, 1-12.

Huber, J. D. y Kleinplatz, P. (2002). Sexual Orientation Identification of men who have sex with men in public settings in Canada. Journal of Homosexuality, 42 (3):1-20.

Humphreys, L. (1970). The Tearoom Trade: Impersonal Sex in Public Places. London: 
Enlarged Edition.

Kernberg, O. F. (1992). La agresión en las perversiones y en los desórdenes de la personalidad. Buenos Aires: Editorial Paidós.

Laguarda Ruíz, R. (2010). El ambiente: espacios de sociabilidad gay en la ciudad de México, 1968-1982. Secuencia. 78, septiembre-diciembre 2010.151-174.

Laguna Maqueda, Ó. E. (2019). Placeres homoeróticos y continuidad de las prácticas vinculadas a la masculinidad hegemónica: el caso de los clientes de bares de estríperes para varones. RELIES, 1(5): 68-89.

Lancaster, R. (1992). Life is Hard. Machismo, Danger and the Intimacy of Power in Nicaragua, Berkley: University of Chicago Press.

Langarita Adiego, J. A. (2013). Sexo sin palabras. La función del silencio en el intercambio sexual anónimo entre hombres. Revista de Antropología Social 22: 313-333.

Langarita Adiego, J. A. (2013a). Ciudad, sexo y estigma. Una aproximación a la práctica del sexo anónimo entre hombres en espacios públicos. Valcuende del Río, J. et. al. (Coords.) Diversidad sexual en Iberoamérica. Sevilla: Aconcagua.

Langarita Adiego, J. A. (2014). Intercambio sexual anónimo en espacios públicos. La práctica del Cruising en el parque de Montjuïc, Gavá y Sitges. Tesis doctoral, Facultad de Geografía e Historia, Universitat de Barcelona.

Langarita Adiego, J. A. (2014a). Sexo y anonimato. Notas sobre los participantes en encuentros sexuales entre hombres en espacios públicos. Revista de Dialectología y Tradiciones Populares. 2:349-368.

Langarita Adiego, J. A. (2014b). Rituales de interacción sexual entre hombres. Una propuesta de análisis del discurso y de la práctica del sexo anónimo. Gazeta de Antropología 30 (3):1-15.

List Reyes, M. (2003). Baños públicos de Puebla, Comunicación presentada al VII Encuentro de Investigadores de Culturas y Ciudades Contemporáneas, México, 10 de septiembre.

Lores, F. (2012). Deseo y placer: anotaciones anotaciones antropológicas a una teoría de la contaminación y de los cuidados sexuales. Tesis doctoral. Facultad de Ciencias Políticas y Sociología. Universidad Complutense de Madrid.

Lozano, I. (2016). Efectos de la homofobia internalizada en la salud mental y sexual de hombres gay en la Ciudad de México. Revista género y salud en cifras 14 (3):32-45.

Mas Grau, J. y Langarita Adiego, J. A. (2015). En tu árbol o en el mío. Una aproximación etnográfica a la práctica del sexo anónimo entre hombres. Revista de Antropología Iberoamericana 10, 2015:289-292.

Mason, G. (2001). Not Our Kind of Crime. Law and Critique,12:252-278.

Norbert, E. (1999). Sociología fundamental. Barcelona: Gedisa.

Núñez Noriega, G. (2001). Reconociendo los placeres, deconstruyendo las identidades. Antropología, patriarcado y homoerotismo en México. Desacatos, primavera 2001:15-34.

Páez Casadiegos, Y. (2006). El escollo de la perversión una genealogía de la estructura perversa. Aposta 30, Julio-Septiembre 2006:1-15.

Pecheny, M. (2001). De la "no-discriminación" al "reconocimiento social". Un análisis de la evolución de las demandas políticas de las minorías sexuales en América Latina. XXIII Meeting of Latin American Studies Association: Washington, DC.

Ponte, M. R. (1974). Life in a parking lot: An ethnography of a Homosexual Drive-in. 
Jacobs, J. (Ed.) Deviance: Field studies and self-Disclosures. California: National Press Book.

Reece, M. y B. Dodge (2004). Exploring the physical, mental and social Well-Being of Gay and Bisexual Men who Cruise for sex on a College Campus. Journal of Homosexuality 46: 111-136.

Rich, A. (1993). Compulsory Heterosexuality and Lesbian Existence. The Lesbian and Gay Studies Reader. New York: Routledge.

Rodríguez Sánchez, N. (2018). De Cuauhtemotzin a las cervecerías. El control oficial del homoerotismo masculino y la construcción estratégica de la geografía disidente, ciudad de México 1930-1951. Historia de Méxicana 68, julio-septiembre 2018:111-175.

Rubin, G. (1984). Reflexionando sobre el sexo: notas para una teoría radical de la sexualidad. Placer y peligro. Explorando la sexualidad femenina. Madrid: Talasa.

Salazar Barrón, S. (2016). La ciudad y el género: la producción urbana del espacio heterosexual. Bitácora arquitectura 33:98-103.

Sánchez Crispín, Á. y Á. López López (2000). Visión geográfica de los lugares gay de la ciudad de México. Cuicuilco 7:1-16.

Sánchez Domínguez, L. A. (2001). Ámbito público y nuevas identidades homosexuales en la Ciudad de México. Cuicuilco 7 (22):81-107.

Sánchez Domínguez, L. A. (2002). De "san juaneras” y “metreras”, entornos públicos y placer homosexual. Memoria 155:25-29.

Teutle, A. y M. List Reyes (2015). Húmedos placeres. Sexo entre varones en saunas de la ciudad de Puebla. México: La Cifra Editorial.

Tewksbury, R. (1996). Cruising for Sex in Public Places: The Structure and Language of Men's Hidden, Erotic Worlds. Deviant Behavior, 17 (1): 1-19.

Troiden, R. R. (1974). Homosexual encounters in a Higway rest stop. E. Goode E. y. Troiden, R. R. (Eds.). Sexual Deviance and Sexual deviant. Nueva York: William Morrow and company.

Turner, V. (1980). La selva de los símbolos. Madrid: Siglo XXI, 2005.

Vázquez García, F. (2001). El discurso médico y la invención del homosexual (España 1840-1915). Asclepio LIII-2:143-162.

Villaamil Pérez, F. y Jociles Rubio, M. I. (2008). Diferencias y desigualdades entre los HSH usuarios de locales comerciales de encuentro sexual: algunas contribuciones a las estrategias comunitarias de prevención del VIH. Etnográfica. 12 (2):185-321.

Villaamil Pérez, F. y Jociles Rubio, M. I. (2011). Risk and community: The impact of HIV among gay in Madrid. The case of sex clubs. Sexualities, 14 (5):580-596.

Williams, W. (1986). The Spirit and the Flessh: Sexual Diversity in American Indian Culture. Boston: Eacon Press.

Wittig, M. (1992). The Straight Mind and Other Essays. Boston: Beacon Pres. 